The next question is, what multiples of these units we should take to make the practical units. In accordance with your request $I$ give my ideas on the subject, premising, however, that $I$ think there is no finality in things of this sort.

First, if we let the rational practical units be the same multiples of the "absolute" rational units as the present practical units are of their absolute progenitors, then we would have (if we adopt the centimetre, gramme, and second, and the convention that $\mu=\mathrm{I}$ in ether)

$$
\begin{aligned}
& {\left[\mathrm{R}_{r}\right] \times 10^{9}=\text { new ohm }=x^{2} \text { times old }} \\
& {\left[\mathrm{L}_{r}\right] \times 10^{9}=\text { new mac }=x^{2}, ",} \\
& {[\mathrm{~S},] \times 10^{-9}=\text { new farad }=x^{-2},,,} \\
& {\left[\mathrm{C}_{r}\right] \times 10^{-1}=\text { new amp }=x^{-\mathrm{I}},,,} \\
& {[\mathrm{V},] \times 10^{8}=\text { new volt }=x,,,} \\
& 10^{7} \mathrm{ergs}=\text { new joule }=\text { old joule. }
\end{aligned}
$$$$
10^{7} \text { ergs per sec }=\text { new watt }=\text { old watt }
$$

I do not, however, think it at all desirable that the new units should follow on the same rules as the old, and consider that the following system is preferable :-

$$
\begin{aligned}
{\left[\mathrm{R}_{r}\right] \times 10^{8} } & =\text { new ohm }=\frac{x^{2}}{10} \times \text { old ohm. } \\
{\left[\mathrm{L}_{r}\right] \times 10^{8}=\text { new mac } } & =\begin{array}{l}
x^{2} \\
\text { 10 }
\end{array} \text { old mac. } \\
{\left[\mathrm{S}_{r}\right] \times 10^{-8} } & =\text { new farad }=\frac{10}{x^{2}} \times \text { old fararl. } \\
{\left[\mathrm{C}_{r}\right] \times \mathrm{I} } & =\text { new amp }=\frac{10}{x} \times \text { old amp. } \\
{\left[\mathrm{V}_{r}\right] \times 10^{8} } & =\text { new volt }=x \times \text { old volt. } \\
\mathrm{IO}^{8} \mathrm{ergs} & =\text { new joule }=10 \times \text { old joule. } \\
10^{8} \mathrm{ergs} \text { per sec. } & =\text { new watt }=10 \times \text { old watt. }
\end{aligned}
$$

It will be observed that this set of practical units makes the ohm, mac, amp, volt, and the unit of elastance, or reciprocal of permittance, all larger than the old ones, but not greatly larger, the multiplier varying roughly from $I_{4}^{1}$ to $3 \frac{1}{2}$.

What, however, I attach particular importance to is the use of one power of 10 only, viz. $10^{8}$, in passing from the absolute to the practical units; instead of, as in the common system, no less than four powers, $10^{1}, 10^{7}, 10^{8}$, and $10^{9}$. I regard this peculiarity of the common system ai a needless and (in my experience) very vexatious complication. In the $10^{8}$ system I have described, this is done away with, and still the practical electrical units keep pace fairly with the old ones. The multiplication of the old joule and watt by to is, of course, a necessary accompaniment. I do not see any objection to the change. Though not important, it seems rather an improvement. (But transformations of units are so treacherous, that I should wish the whole of the above to be narrowly scrutinized.)

It is suggested to make $10^{9}$ the multiplier throughout, and the results are :- -

$$
\begin{aligned}
& {\left[\mathrm{R}_{r}\right] \times 10^{9}=\text { new ohm }=x^{2} \times \text { old ohm. }} \\
& {\left[\mathrm{L}_{r}\right] \times 10^{9}=\text { new } \mathrm{mac}=x^{2} \times \text { old mac. }} \\
& {\left[S_{r}\right] \times 10^{m 9}=\text { new farad }=x^{m-2} \times \text { old farad. }} \\
& {[\mathrm{C},] \times \mathbf{r}=\text { new } \mathrm{amp}=\frac{\mathrm{IO}}{x} \times \text { old amp. }} \\
& {\left[\mathrm{V}^{r}\right] \times 10^{9}=\text { new volt }=\text { IO } x \times \text { old volt. }} \\
& 10^{9} \text { ergs }=\text { new joule }=10^{-2} \times \text { old joule. } \\
& 10^{9} \text { ergs p. sec. }=\text { new watt }=10^{2} \times \text { old watt. }
\end{aligned}
$$

But I think this system makes the ohm inconveniently big, and has some other objections. But I do not want to dogmatize in these matters of detail. Two things I would emphasize :First, rationalize the units. Next, employ a single multiplier, as, for example, $10^{8}$.

\section{Oliver Heaviside.}

P.S.-Heaven preserve us from dynamics based on the Act of Parliament !

\section{Neutral Point in the Pendulum.}

IN the theory of the pendulum the position of the neutral point of support is a matter of practical importance, which is, nevertheless, quite disregarded.

Taking a rigid uniform bar as the simplest case, there are NO. 1187 , VOL. 46] four points of support from which its vibrations are equal, the two ends and the two respective centres of oscillation. But there are two symmetric points, situated between either end and the centre of oscillation nearest to that end, from which points of suspension the rate of vibration is most rapid. Hence, when suspended from these points, a change in the position of the point of support produces a minimum difference in the rate of vibration. Or, in practical terms, there is a great advantage in having a small amount of overhead weight above the support, as then, if the support approach the bob (owing to changes in elasticity of the spring, or of the knife edges), and so increase the number of vibrations, it recedes from the top weight, and so diminishes the vibrations to a corresponding amount, and vice versa.

This neutral point of support seems to have been overlooked in the main pendulum researches, as it was what had to be avoided rather than sought in the determination of the length, which was then the main interest. Probably some one has already noticed such an elementary property; but it is of so much value in minimizing sources of error that it is worth some attention.

Bromley, Kent.

W. M. Flinders Petrie.

\section{Induction and Deduction.}

CAN we determine the precise relation between Induction and Deduction? Both are said to be a species of Inference. Deauction is, no doubt, Mediate Inference. Is Induction Mediate or Immediate Inference? If Immediate it must be of the form :

This X is Y (or these X's are Y's) . . . . . (1)

$\therefore$ All X's are Y's .......... (2)

But such "inference" as this is not illative; (I) can furnish only a suggestion, not by any means a justification, of $(2)$.

Still it is true that if, e. $\rho$. I have proved that the angles at the base of an isosceles triangle are equal to each other, I henceforth believe and assert unhesitatingly, that all isosceles triangles have the angles at the base equal. How do I justify such a conclusion of an universal from a particular? In this way, I think :-Every nameable or cogitable object is an identity in diversity - that is, it is itself, it is something, and it has a plurality of characteristics. This principle is involved in the assertion of any statement of the form $A$ is $\mathrm{B}$, and it seems moreover to be, in itself, evident on reflection. Further (as Bacon surmised), every property (or group of properties; has a "form," some invariable and inevitable coexistent. In other words, there is uniformity of coexistence as well as of causation in nature. In the case of any one isosceles triangle, I have seen the connection of interdependence that there is between the characteristics of "having equal sicles," and "having the angles at the base equal ;" I have perceived it to be self-evident that the one property involves the other. Hence, my whole argument might run thus :-

Every characteristic is invariably accompanied by some other characteristic

Equality of sides in a triangle is a characteristic ;

$\therefore$ Equality of sides in a triangle is invariably accompanied by some other characteristic.

Again :-

Equality of angles at the base is a characteristic which is (selfevidently) inseparable from equality of sides in one [this particular] case ;

What is in eparable from equality of sides in one case is in separable in all cases ;

$\therefore$ Equality of angles at the base is inseparable from equality of sides in all cases-

That is, all isosceles triangles have the angles at the base equal.

What we rely on here is Interdependence of characteristics and Uniformity of that interdependence; i.e. we rely on a principle of coexistence or coinherence, parallel to Mill's "Law of Causation"; and this is a principle which we find to be a necessary condition of what we accept as strictly self-evident propositions. The assertion with which we conclude in the above generalization, is an assertion of uniformity of interdependence between certain specified characteristics.

Again, if $I$ administer a certain amount of arsenic to a healthy animal, and it dies, and I hence conclude that arsenic is a cause 